Article

\title{
Genome-Wide Identification of PIFs in Grapes (Vitis vinifera L.) and Their Transcriptional Analysis under Lighting/Shading Conditions
}

\author{
Kekun Zhang, Ting Zheng, Xudong Zhu®, Songtao Jiu, Zhongjie Liu, Le Guan, Haifeng Jia \\ and Jinggui Fang * \\ College of Horticulture, Nanjing Agricultural University, Nanjing 210095, Jiangsu, China; \\ zhangkekun1990@163.com (K.Z.); 2016204006@njau.edu.cn (T.Z.); 2014204002@njau.edu.cn (X.Z.); \\ 2015204003@njau.edu.cn (S.J.); 2017204011@njau.edu.cn (Z.L.); 2017104011@njau.edu.cn (L.G.); \\ 2014002@njau.edu.cn (H.J.) \\ * Correspondence: fanggg@njau.edu.cn; Tel.: +86-025-8439-5217
}

Received: 2 August 2018; Accepted: 3 September 2018; Published: 7 September 2018

\begin{abstract}
Phytochrome-interacting factors (PIFs), as the basic helix-loop-helix (bHLH) transcription factors, are the primary signaling partners for phytochromes (PHY) that play a key role in PHY-mediated light signal transduction. At present, there are few studies on PIFs in fruit trees. In order to clarify the status of PIFs in grapevines, we identified members of the grape PIFs family and conducted phylogenetic and expression analysis. We identified PIF1, PIF3, PIF4, and PIF7 in PIFs families of the grapevine (Vitis vinifera L.), which were distributed on four different chromosomes with similar gene structures. Except for the closer relationship with PIF1 of citrus, PIFs of grape were distant from the other fruit species such as apple, pear, peach, and strawberry. The VvPIFs (except VvPIF4) were located in the syntenic block with those from Arabidopsis thaliana, Solanum lycopersicum, or Citrus sinensis. In addition to PIF1, all PIFs in grapevines have conserved active PHYB binding (APB) sequences. VvPIF1 has a conserved PIF1-specific active PHYA binding (APA) sequence, while amino acid mutations occurred in the specific APA sequence in VvPIF3. Interestingly, two specific motifs were found in the PIF4 amino acid sequence. The photoreceptor-related elements in the VvPIFs promoter region were the most abundant. PIF1, LONG HYPOCOTYL 5 (HY5) and PIF3, PIF4, GIBBERELLIC ACID INSENSITIVE 1 (GAI1) may interact with each other and participate together in light signal transduction. The relative expression levels of the VvPIFs showed diverse patterns in the various organs at different developmental stages, of which PIF4 was most highly expressed. Prior to maturation, the expression of PIF4 and PIF7 in the skin of the different cultivars increased, while the expression of all PIFs in the flesh decreased. The transcription level of PIFs in grape leaves was sensitive to changes in lighting and shading. Shading treatment was beneficial for enhancing the transcription level of VvPIFs, but the effect on VvPIF3 and VvPIF4 was time-controlled. We concluded that PIFs in grapevines are both conservative and species-specific. The identification and analysis of grape PIFs could provide a theoretical foundation for the further construction of grape light regulation networks.
\end{abstract}

Keywords: PIFs; grapes; phylogenetic analysis; gene expression; lighting and shading conditions

\section{Introduction}

Plants undergo the developmental program of photomorphogenesis in the presence of light [1]. Phytochromes, acting as red/far red-light sensors, are one of the most important photoreceptors in plants, and have been investigated extensively in terms of photochemistry, protein structures, and 
transduction of light signal [2,3]. There are various types of phytochromes in plants. PHYA, $P H Y B$, PHYC, PHYD, and PHYE were reported in Arabidopsis thaliana, PHYA, PHYB, PHYC in Oryza sativa, PHYA1, PHYB1, PHYC1 and their homologous genes PHYA2, PHYB2, PHYC2 in Zea mays [1]. PHYs exist in both $\mathrm{P}_{\mathrm{r}}$ and $\mathrm{P}_{\mathrm{fr}}$ forms. Red-light activates PHYs by converting the $\mathrm{P}_{\mathrm{r}}$ form to the $\mathrm{P}_{\mathrm{fr}}$ form, whereas far red-light inactivates $P_{f r}$ by converting it back to the $P_{r}$ form.

Phytochrome-interacting factors (PIFs), the primary signaling partners for phytochromes, are basic helix-loop-helix (bHLH) transcription factors that play a key role in PHY-mediated light signal transduction. Similar to phytochromes, PIFs have been discovered in a variety of plant lineages from bryophytes to angiosperms [4,5]. In various plants, there are many endogenous (gibberellins, auxin, ethylene), environmental (blue light, high temperature) and developmental signaling pathways (circadian clock, cell fate) intersecting with PIF activity in regulating various morphogenic responses [6].

In Arabidopsis, many PIFs have been identified and functionally characterized. PIF1 has been shown to play a major role in inhibiting light-dependent seed germination [7], and regulates key genes involved in chlorophyll biosynthesis to optimize the greening process in direct or indirect ways [8]. PIF3 could promote hypocotyl elongation in response to ethylene and the biosynthesis of chlorophyll and anthocyanin accumulation under light conditions [9], negatively regulates seedling de-etiolation along with other PIFs [10,11], and recently, PIF3 was also shown to modulate the freezing tolerance as a negative regulator of the expression of $C$-repeat binding factor $(C B F)$ genes [12]. PIF4 regulates the stomatal development in response to different light qualities, chlorophyll degradation and leaf senescence in darkness, and the freezing tolerance of the plant [13-15]. Additionally, it can uniquely regulate hypocotyl elongation in response to light and diurnal conditions, and early flowering response to high temperature $[16,17]$. PIF5 negatively regulates the red-light-induced anthocyanin biosynthesis and participated in many pathways regulated by PIF4 [18]. PIF6 has two splice variants, $a$ and $b$, of which the b-form participates in the regulation of seed dormancy [19]. PIF7, as an interactor of PHYB, regulates the shade avoidance responses by allowing its dephosphorylated form to bind to the auxin biosynthetic genes [20]. In the meanwhile, many PIFs could coordinate to modulate certain physiological responses. Leivar et al. [21] found that PIF7 could interact with PIF3 and PIF4 to maintain the low levels of $\mathrm{PHYB}$, involved in the process of the seedlings de-etiolation under far red-light. PIF3 and PIF4 interact with BRASSINAZOLE-RESISTANT 1(BZR1) to regulate genes involved in the brassinosteroid signaling pathways by binding to the same G-box element [22,23]. PIF1 also negatively regulates both biosynthesis and accumulation of carotenoids coupled with PIF3 [24].

At present, PIFs have been widely studied in Arabidopsis, tomato, maize, rice and other herbs [1,25-27], but little in woody plants. Grape is an important fruit tree resource in the world. The members of the PIFs gene family in grapes have not yet been identified and their function in grapevine photomorphogenesis, fruit development, regulation of endogenous metabolism, and reaction to external environment remains to be studied.

In this study, we identified a total of four PIF genes in the grape using AtPIFs as a template. Afterwards, we carried out the gene structure analysis of the PIFs in the grape, other five herbaceous plants and three woody plants, and analyzed the amino acid conserved functional domains and the cis-elements in the promoters. To further clarify the function of those genes in grapes, we also studied the transcriptional levels of PIFs in different organs of grapevines, the sensitivity of VvPIFs to different stresses in the berries and leaves. The results of our study can provide theoretical support for the further construction of regulation network of PIFs in grape and other fruit trees.

\section{Methods}

\subsection{Plant Materials and Treatments}

\subsubsection{Grape Berries in Development}

The 6-year-old grape varieties Summer black (SB) and Shine Muscat (SM) planted in the Jiangsu Academy of Agricultural Sciences were used as materials to study the transcription patterns of PIFS 
during grape berry development. Twenty vines of each variety were chosen and the berry samples (three biological replicates) were collected at the following four developmental stages (DS): (i) DS1, the early stage of fruit enlargement (20 days after anthesis); (ii) DS2, pre-verasion (10\% of the berries began to change color); (iii) DS3, post-verasion ( $90 \%$ of the berries completed the color changing); and (iv) DS4, maturity (the content of total soluble solids (TSS) and titratable acid (TA) tended to be stable). Samples were randomly collected, and the location of vines and clusters were also considered. Young leaves, young tendrils, young stems, young roots were also sampled for tissue-specific expression analysis. Mature berries were separated into the skin and flesh, and then were immediately frozen in liquid nitrogen individually. All samples were stored at $-80^{\circ} \mathrm{C}$ for the subsequent analysis.

\subsubsection{Grape Leaves Used for Lighting/Shading Studies}

Potted 2-year-old Summer Black grapes were used for the experiment. The grape seedlings grew in an artificial climate chamber at $25^{\circ} \mathrm{C}$ overnight before the treatment. Tinfoil paper with no light transmittance was used as the light-shielding material, and the leaves at the 4th to 6th nodes were selected for testing. Half the blades are treated with light and half are shaded along the dividing line of the main vein. One control (normal illumination, Control Check (CK)) and two treatments were set in the test. One treatment was a shading treatment, and the other was a light treatment after $6 \mathrm{~h}$ of shading. The sampling time started from shading and was set at $0 \mathrm{~h}, 2 \mathrm{~h}, 4 \mathrm{~h}, 6 \mathrm{~h}, 8 \mathrm{~h}, 10 \mathrm{~h}, 12 \mathrm{~h}$ after shading. The collected leaves were immediately frozen in liquid nitrogen and stored at $-80^{\circ} \mathrm{C}$.

\subsubsection{Grape Berry Samples Used for Lighting/Shading Studies}

The 6-year-old grape varieties Summer Black in Wuhu, Anhui Province, China were tested. 20 bunches of grape berries were shaded with tinfoil paper and another 20 bunches without shading. Fruit bagging was performed 30 days after fruit setting, and samples were taken at 0 days (d), $15 \mathrm{~d}$, and $30 \mathrm{~d}$ after bagging. The berry sample was collected and stored as described above.

\subsection{Genome-Wide Identification and Annotation of Grape PIFs Genes}

We downloaded the protein sequence of Arabidopsis PIFs from the TAIR Arabidopsis database [28] and then blasted the homologous sequence against the grape genome database. To obtain a precise list of grapevine PIFs genes, we searched and downloaded the annotated grapevine proteins from three public databases: the National Centre for Biotechnology Information (NCBI; http:/ / www.ncbi.nlm. nih.gov /), the Genoscope [29] and the Grape Genome Database [30,31]. The choice of the candidate PIF was based on the E-value $\left(1 \mathrm{e}^{-5}\right)$ and the highest similarity scores. All the obtained sequences were stored in the InterProScan database [32] and the Conserved Domains Database [33] to confirm their completeness and existence of the core domains. Length of sequences, molecular weights and isoelectric points of deduced polypeptides were calculated by using tools provided at the ExPasy website [34,35]. The choice of candidate PIFs in Brassica napus, Nicotiana tabacum, Solanum lycopersicum, Prunus persica, Malus domestica, Pyrus bretschneideri, Citrus sinensis, Fragaria ananassa was also based on the E-value $\left(1 \mathrm{e}^{-5}\right)$ and the highest similarity scores to AtPIFs in NCBI.

\subsection{Gene Structure, Phylogenetic, Conserved Motifs and Syntenic Analysis of PIFs Family}

Exon and intron structures of the VvPIFs were determined based on their coding sequence (CDS) and the correspondent full-length gene sequences in NCBI. The gene structures were thereafter illustrated by the online program Gene Structure Display Server [36]. MEGA version 6 (Sudhir Kumar, Arizona State University, Temp, AZ, USA) was used to construct phylogenetic trees by the Maximum Likelihood (ML) methods and the bootstrap test carried out with 1000 replicates [37]. The conserved motifs were identified using the online MEME program (version 4.12.0) [38]. We set the motif number as 10 and chose motifs with E-values $\leq 1 \mathrm{e}^{-30}$.

MCscan X (Yupeng Wang, University of Georgia, Athens, GA, USA) was used to analyze the gene synteny and collinearity of PIFs among Vitis vinifera, A. thaliana, S. lycopersicum, and C. sinensis [39]. 
The synteny figures were drawn by Circos-0.69 [40] and those results with E-value $>1 \mathrm{e}^{-5}$ were filtered.

\subsection{Multiple Sequence Alignments, Promoter Analysis and Interaction Protein Prediction}

Multiple sequence alignments of PIFs were performed using the MEGA version 6 [37]. The $1500 \mathrm{bp}$ upstream of the PIFs genes of each species was used to perform cis-elements analysis in PlantCARE [41]. The protein sequences of VvPIFs were downloaded and used for interaction analysis and prediction in the online software STRING (ELIXIR, Hinxton, UK, https: / / string-db.org).

\subsection{Transcriptome and Microarray Data Acquisition and Analysis}

The transcriptome data of organs such as roots, stems, leaves, flowers, berries, and tendrils are from Gene Expression Omnibus (GEO, available online: https://www.ncbi.nlm.nih.gov/geo/). Data code was GSE36128 and the chosen cultivar was Corvina. The short term abiotic stress data was from GSE31594, in which Cabernet Sauvignon was treated with $120 \mathrm{mM}$ salt $(10: 1 \mathrm{NaCl}: \mathrm{CaCl})$, Polyethylene Glycol, cold $\left(5^{\circ} \mathrm{C}\right)$ or unstressed. The long-term salt and water stress data was from GSE31677, where the potted Cabernet Sauvignon vines in the greenhouse were exposed to irrigated controls, non-irrigated water-deficits, and saline treatments for 16 days. The expression data were normalized according to Zhu et al. [31]. The transcriptome data for strawberry, citrus, and apple fruits during their developmental period were also downloaded from the GEO database and numbered GSE85572, GSE69432, and GSE64079.

\subsection{Total RNA Isolation, cDNA Synthesis and Gene Expression Analysis}

Total RNAs were isolated using a CTAB method according to Wang et al. [42]. Then the first strand cDNA was synthesized from $1 \mu \mathrm{g}$ total RNA with P1 [an oligo(dT) ${ }_{20}$ primer], P02 (a random primer) and SuperscriptIII RNase H-RT kit from Invitrogen (Carlsbad, CA, USA) according to the manufacturer's instruction. The cDNA was diluted at 1:10 for RT-PCR.

Real time-PCR (RT-PCR) was carried out using the CFX96 Real-Time PCR Detection system (Bio-Rad, Hercules, CA, USA). Reaction mixes volume was $10 \mu \mathrm{L}$, which included $5 \mu \mathrm{L}$ of SYBR Green Supermix (Bio-Rad), $2 \mu \mathrm{L}$ of diluted cDNA, and $0.2 \mu \mathrm{L}$ of each primer. Each pair of quantitative real time PCR (qRT-PCR) primers were validated by cloning and sequencing of the RT-PCR product with this pair of primers. The efficiency of each primer pair was quantified using a PCR product serial dilution. All biological samples were assayed in technical triplicates. Ubiquitin and EF1 $\gamma$ were used as internal standards and for normalizing the expression. Expression levels were calculated based on the $2^{-\Delta \mathrm{Ct}}$ method. Due to the low expression values of PIFs in some organs of the grape berries, expression levels in those cases were calculated based on the $2^{-\Delta \Delta \mathrm{Ct}}$ method with the first stage sample chosen as a reference for convenience of observation.

\subsection{Statistical Analysis}

Statistical analysis was performed with the software SAS 9.2 (SAS Institute Inc., Cary, NC, USA). Differences between genotypes and tissues for a giving sampling date were analyzed by a two-way analysis of variance (ANOVA) followed by the Duncan's multiple comparison test at $p<0.05$. Correlation network analysis was conducted in the Heml1.0.3.7 software (Yu Xue, Huazhong University of Science and Technology, Wuhan, China) and the line chart was performed by Origin 2017 (OriginLab Corporation, Northampton, MA, USA).

\section{Results}

\subsection{Identification of Grape PIFs}

A total of 4 PIF members were identified in the three grape genome database [29-31]. The four members were named as PIF1, PIF3, PIF4, and PIF7, respectively and their corresponding 
serial numbers are LOC100264869, LOC100247781, LOC100262490, and LOC100262138 in NCBI, VIT_07s0005g05100, VIT_14s0060g00260, VIT_12s0028g01110, and VIT_17s0000g06930 in CRIBI, and GSVIVT00028516001, GSVIVT00031338001, GSVIVT00020814001, and GSVIVT00007914001 in the 12X grapevine genome database. The basic information of the four genes was listed in Table 1. It can be seen that the gene length of the four PIFs varied from 3617 to $5463 \mathrm{bp}$, and the length of amino acids sequence ranged from 423 to 709 . PIF3 and PIF7 have the largest or the smallest genomic DNA, number of amino acids and protein molecular weight individually. The predicted isoelectric point range of various proteins ranged from 5.32 to 8.94, indicating that those proteins might function in different subcellular compartments. What is more, each protein contains a bHLH domain.

Table 1. Characteristics of Phytochrome-Interacting Factors (PIFs) genes.

\begin{tabular}{|c|c|c|c|c|c|c|c|}
\hline Name & Locus Id & $\begin{array}{c}\text { Genomic } \\
\text { DNA Size } \\
\text { (bp) }\end{array}$ & $\begin{array}{l}\text { Number of } \\
\text { Amino Acids }\end{array}$ & $\begin{array}{l}\text { Predicted } \\
\text { Mw (kDa) }\end{array}$ & $\begin{array}{c}\text { Theoretical } \\
\text { pI }\end{array}$ & $\begin{array}{l}\text { Chromosome } \\
\text { Location }\end{array}$ & $\begin{array}{c}\text { Functional } \\
\text { Domains } \\
\text { (Start-End, bp) }\end{array}$ \\
\hline PIF3 & LOC100247781 & 5463 & 709 & 75.861 & 6.3 & 14 & 462-511/bHLH \\
\hline PIF4 & LOC100262490 & 3798 & 531 & 57.765 & 7.3 & 12 & 338-387/bHLH \\
\hline PIF7 & LOC100262138 & 3617 & 423 & 46.631 & 8.94 & 17 & 223-272/bHLH \\
\hline
\end{tabular}

Note: bHLH: basic helix-loop-helix.

As for the comparison among CDS (Table 2), the similarity between each pair was more than $40 \%$, with the highest similarity being between PIF4 and PIF1 (55\%). But for the amino acid sequences, the similarity of each pair was low, ranging from $22 \%$ to $33 \%$.

Table 2. Coding region nucleotide (upper portion of matrix) and amino acid (bottom portion of matrix) sequence pairwise comparisons (\% similarity) between grape PIF genes.

\begin{tabular}{lcccc}
\hline & VvPIF1 & VvPIF3 & VvPIF4 & VvPIF7 \\
\hline VvPIF1 & - & $48 / 29$ & $55 / 19$ & $51 / 24$ \\
VvPIF3 & $26 / 34 / 39$ & - & $50 / 29$ & $44 / 41$ \\
VvPIF4 & $33 / 42 / 24$ & $27 / 38 / 28$ & - & $49 / 26$ \\
VvPIF7 & $28 / 41 / 20$ & $22 / 31 / 41$ & $25 / 35 / 34$ & - \\
\hline
\end{tabular}

Note: This table is divided into two parts, the upper part (upper right corner) representing the nucleotide comparison between different PIFs, and the lower part (lower left corner) representing the comparison of protein sequences between different PIFs. The numbers represent Identities/Positives/Gaps (lower left corner) ratio, and Identities/Gaps (upper right corner) ratio respectively.

\subsection{Phylogenetic, Conserved Structural and Syntenic Analysis of PIFs}

According to the estimated divergence times for the molecular phylogenetic analysis by ML method (timetree) (Figure 1), the PIF family members were roughly divided into 5 categories (the relative divergence time at the branching points was larger than 150). PIF1 located in the first category, while PIF4 and PIF5 in the second, PIF3 in the third, PIF7 and UNE10 in the fourth, and PIF6 in the fifth. In the first category, PIF1 could be divided into two classes, SIPIF1 and NtPIF1-like were clustered together with a far kinship to the others. The fruit species were closely related, in which the kinship between the grape and the citrus was the closest. In the second category, the PIFs could also be broadly divided into two classes. PIF4, PIF5 in the A. thaliana and B. napus were clustered together and other species were clustered in another class. But in the second class, VvPIF4 had a far kinship with other fruit species. In the PIF3, CsPIF3 diverged from other species at the earliest time, and VvPIF3 had a distant relationship with other species. In the PIF7, VvPIF7 was at the starting node of fruit species classification and has a distant relationship with others. PIF6 was only found in A. thaliana and B. napus. Overall, except for the closer relationship with citrus on PIF1, PIFs of grapes were far from the other fruit species such as apples, pears, peaches, and strawberries. 


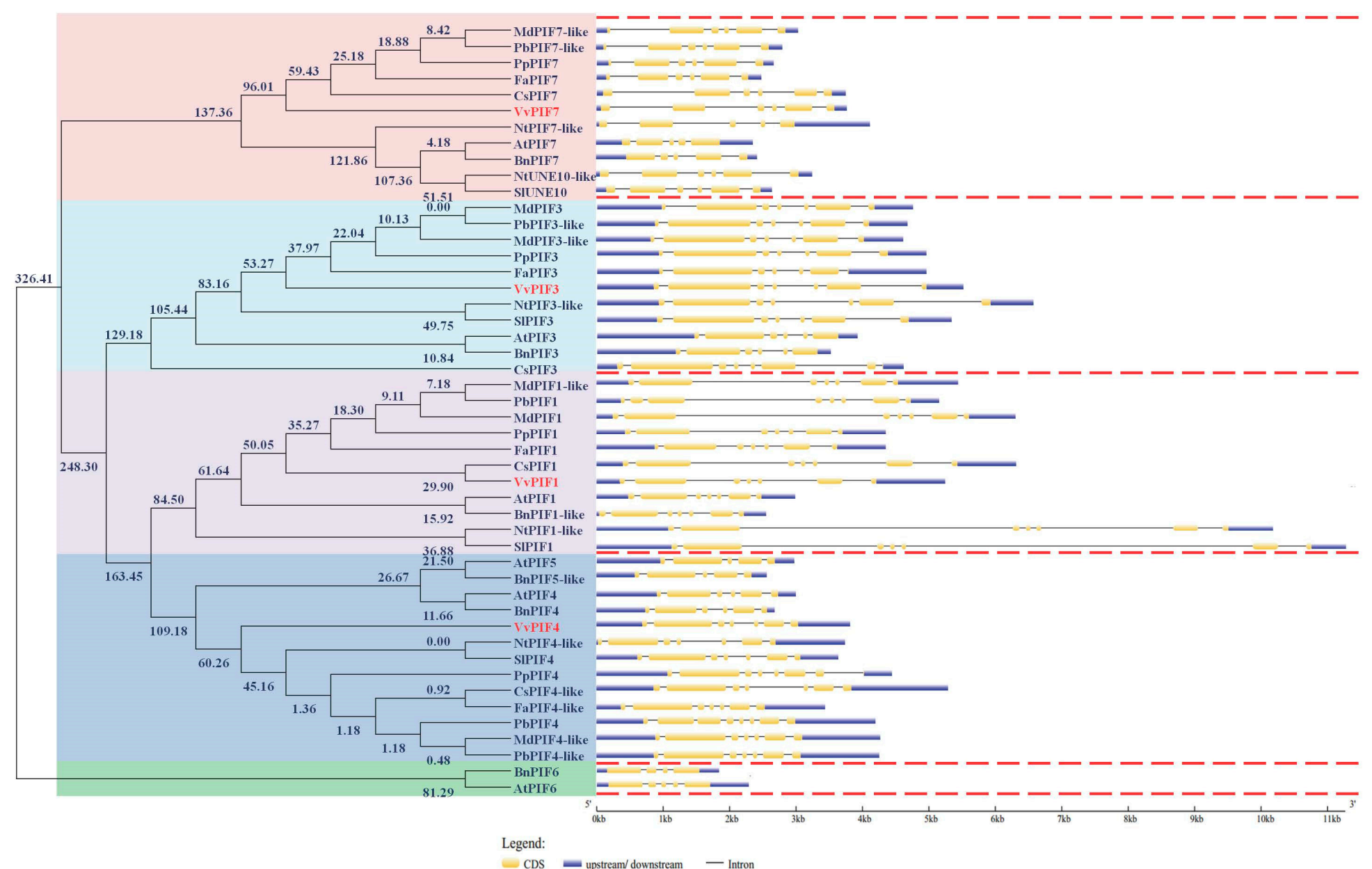

Figure 1. The phylogenetic tree of PIF proteins and their gene structure characteristics. Members of PIFs from the Vitis vinifera (red mark), Arabidopsis thaliana, Brassica napus, Nicotiana tabacum, Solanum lycopersicum, Prunus persica, Malus domestica, Pyrus bretschneideri, Citrus sinensis, and Fragaria ananassa were put together for comparison. The phylogenetic tree was generated by MEGA 6.0 using Maximum Likelihood method (timetree). The axis numbers mean the relative divergence time. CDS: Coding sequence. 
From the gene structure of PIF1 (Figure 1), it could be found that the SIPIF1 was longest, BnPIF1-like was the shortest and the PIF1 of fruit species was of moderate length. Except for PbPIF1, all plants contained seven exons in PIF1. In these exons, the second and the sixth exon were longer, others were shorter and the distance between exon3, 4 and 5 was short. For PIF3, the NtPIF3-like was the longest in sequence length while the BnPIF3 was the shortest. There were seven exons in the PIF3 of woody trees, of which the second and the sixth were longer. For PIF4 and PIF5 structure analysis, BnPIF4 was the shortest and CsPIF4 was the longest. There were seven exons in VvPIF4, with the similar structure to PIF4 in other plants. As for PIF7, there were six exons in VvPIF7. After UTR end removed, VvPIF7 was the longest. In general, the PIF1 gene structure, with the stable number of exons and introns, was relatively conservative, but the gene length varied greatly between different species. The structure of PIF3, PIF4, and PIF7 genes differed among different species.

According to the results of syntenic analysis (Figure 2, Table S1), we found that there were VvPIFs corresponding genes in A. thaliana, S. lycopersicum, and C. sinensis, and these genes were located at the same syntenic block. Combined with the relative evolution time analysis in Figure 1, VvPIF7 evolved earlier, and might be derived from a common ancestor with CSPIF7 and SIUNE10. VvPIF3 evolved later than CSPIF3, and might had a common origin with AtPIF3. VvPIF4 was not located in the syntenic block, and might had certain specificity due to its late evolution time. Arabidopsis, tomato and citrus are important model plants for studying herbaceous plants and fruit trees. The study of the collinear relationship of PIFs in these plants would shed important implications for the functional study of VvPIFs.

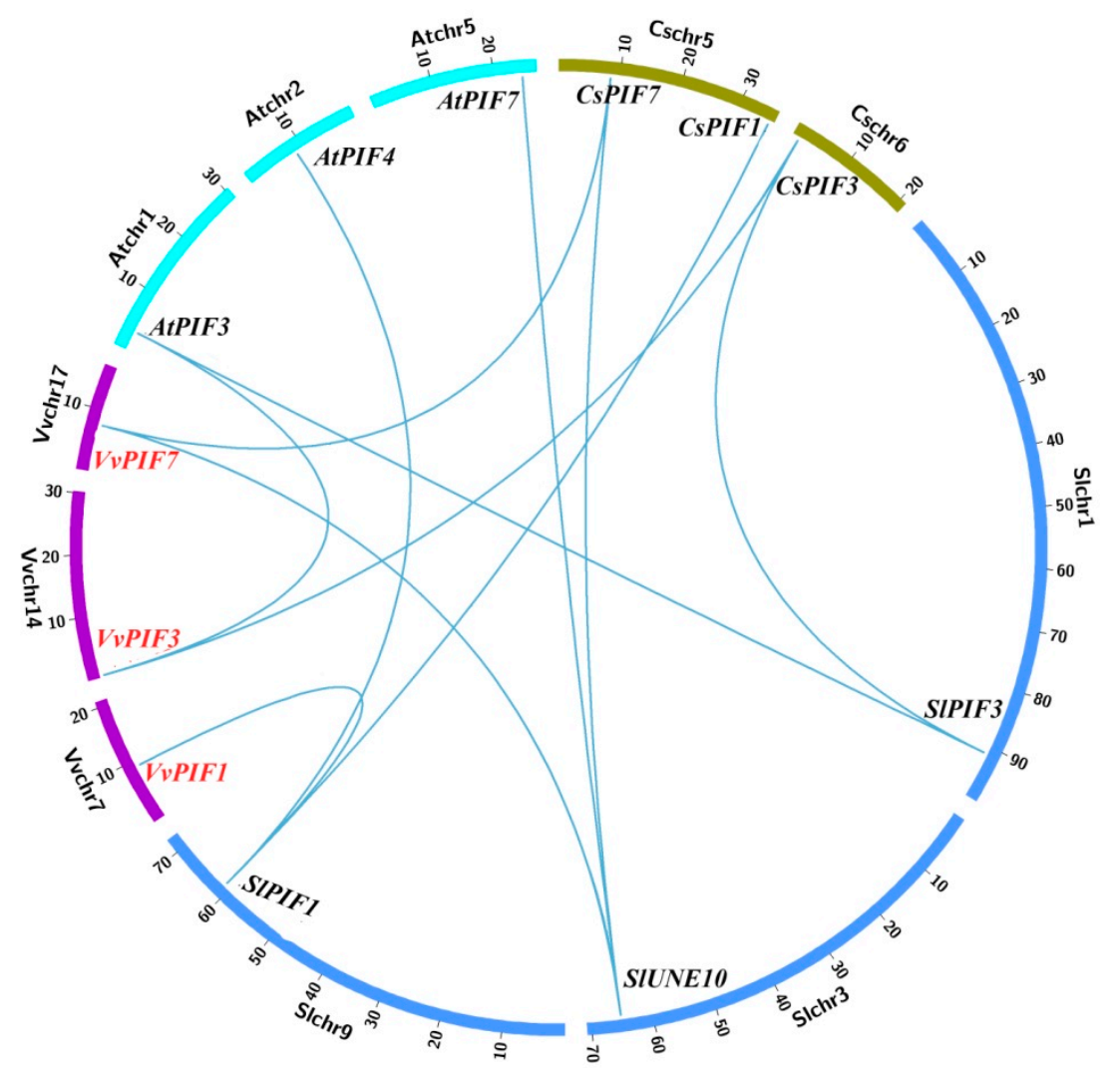

Figure 2. Syntenic block among PIFs from V. vinifera, A. thaliana, S. lycopersicum, and C. sinensis. The chromosomes of $V$. vinifera, A. thaliana, S. lycopersicum, and C. sinensis are displayed in purple, bright green, blue, gray green. The putative orthologous PIFs are connected by light blue.

\subsection{Comparison of Conserved Motifs}

The conserved motifs of active PHYB binding (APB) in A. thaliana were E41, L42, G47, and Q48 [43]; of PIF1- (active PHYA binding) (APA) were L95, N144, and of PIF3-APA were F203, F209 [44]. 
From the Figure 3, it could be found that the motifs of APB in VvPIF1, CsPIF1, MdPIF3, CsPIF4-like, SIPIF4, NtPIF4-like, FaPIF7, PbPIF7-like, MdPIF7-like, NtUNE10-like changed (Figure 3A-D), which might affect their combination with PHYB. The change in the APA conserved sequence of PbPIF1 indicated its poor binding to PHYA. In addition to AtPIF3 and NtPIF3, F209 of PIF3-APA in other plants all mutated to L. Since that F203 and F209 were mutated together in the previous motifs study [44], whether a single mutation occurs in these two sites affects the combination of PHYA with PIF3 remains to be further explored.

After conservative motifs analysis of PIFs family, we obtained 10 conserved domains, of which the second domain contained APB conserved motifs (Figure 4, Figure S1). A total of seven domains were detected in PIF1, and the fourth, fifth, and tenth motifs were missing. The conserved domains of AtPIF1 consisted of only five kinds. Grapes lack the second motif compared to other species, consistent with the results of the previous study (Figure 3). Except for grapes, motifs of other fruit species were similar. What's more, motif 9 was a specific motif in PIF1 compared to other PIFs. Seven conserved motifs were detected in PIF3, these motifs were similar among fruit species, except MdPIF3. A total of 9 conserved motifs were detected in PIF4, and BnPIF4 had the least number with only 4 kinds of motifs. With the exception of PbPIF4, the motifs of other fruit species were similar. Compared with other motifs, motif 5 and motif 10 were specially detected in PIF4 and motif 4 in PIF3 and PIF4. In PIF7, six conserved sequences were detected in grapevines, similar to those in S. lycopersicum and N. tabacum, of which motif 1 and motif 2 existed in all fruit species, but others differed greatly among species. Above all, motif 1, 2, 7, 3, 6, 8 seem to be the conserved motifs of PIFs.

\subsection{Cis-Element Analysis in the PIF Gene Promoters and Functional Prediction of PIFs Proteins}

To further clarify the gene function and transcriptional regulation mechanism of PIFs, we amplified the 1500 bp upstream of the PIFs genes and analyzed their cis-elements. The predicted cis-elements differed among species and genes, but the cis-elements related to photoreaction were the most abundant (ACE, G-Box, GT1-motif, Sp1, ATCT-motif, Box 4, I-box, TCT-motif, AT1-motif), which had the largest number in all species (Figure 5).

The number of cis-elements in the promoters of PIFs was also various, and nine were detected in VvPIF1, 12 in VvPIF3, 7 in VvPIF4, and 11 in VvPIF7. In the promoter of VvPIF1, the number of endosperm expression related elements (Skn-1 motifs) was the largest except those related to photoreaction. In the promoter of PIF3 and PIF7, there was many elements for abscisic acid responsiveness (ABRE), but no for circadian control (circadian: CANNNNATC) in grapes when compared with other fruit species. The cis-elements in the VvPIF4 promoter were less diverse. Compared with other fruit trees, the upstream of VvPIF4 lacked the anaerobic induction (ARE), drought-inducibility (MBS) related elements, and had a seed-specific regulation class (RY-Original) element. Last but not least, there were no low-temperature-responsive elements detected in the promoters of VvPIFs.

As we all know, PIFs are involved in the transduction of light signals in plants. However, the specific regulatory network of PIFs in grapes is still not clear. VvPIF1 could interact with PHYA, PHYE, and HY5 to participate in the regulation of diurnal growth and development of plants (Figure S2, Table S3). VvPIF3 not only combined with photoreceptors, but also interacted with DELLA protein GAI1 to participate in the signal transduction process of plants. PIF4 could also interact with GAI1 to regulate the growth process. In addition to the known phytochromes, hormone signaling-related proteins, etc., PIFs could also interact with many undefined functional proteins, and their metabolic network remained to be further studied. To further clarify the relationship between PIF gene and grape growth, we also performed expression analysis. 


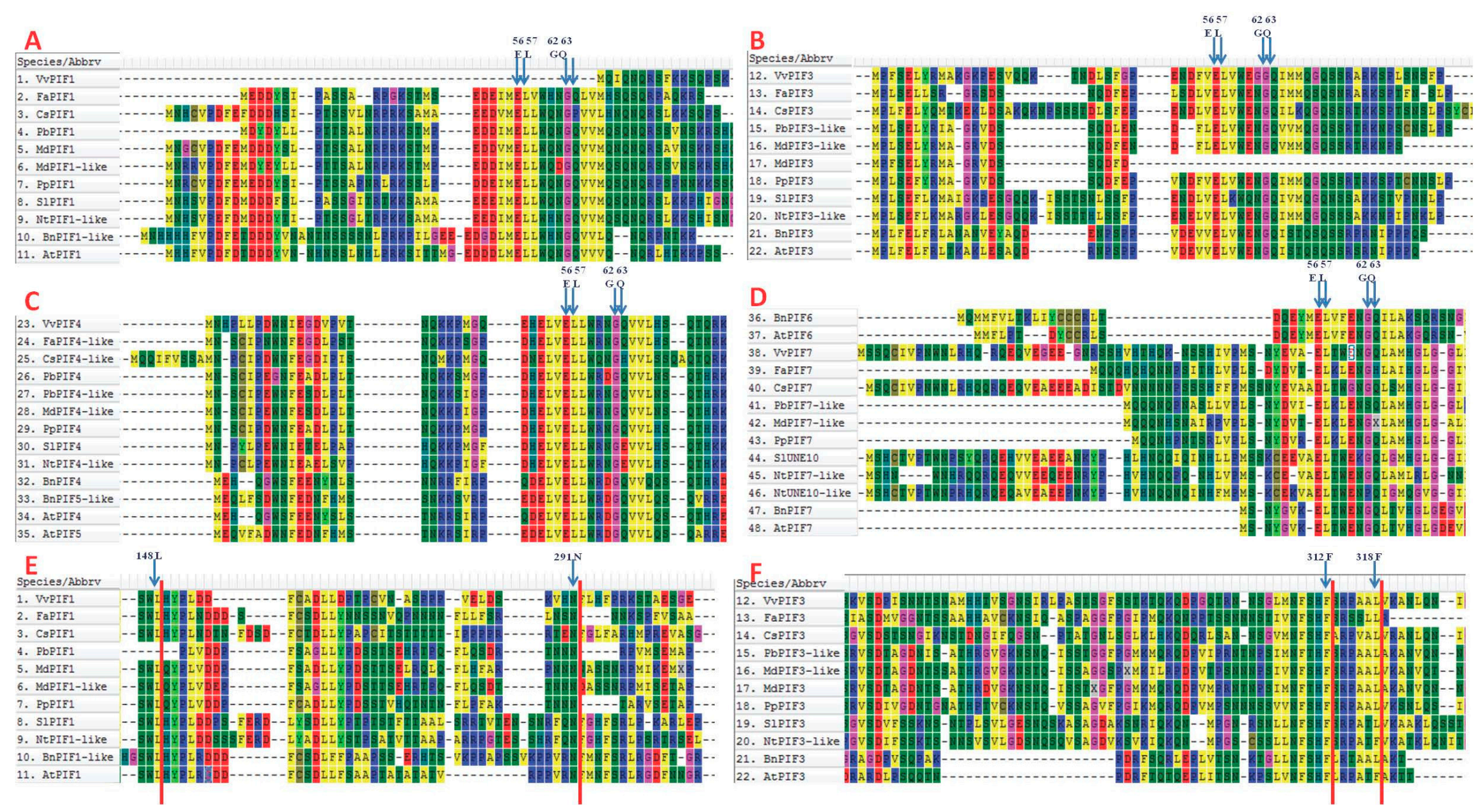

Figure 3. The multiple alignment of deduced amino acid sequences of PIFs for the comparison of active PHYB binding (APB) and active PHYA binding (APA) conserved domains. (A-D) Comparison of APB conserved domains in PIFs; (E) Comparison of APA conserved domains in PIF1; (F) Comparison of APA conserved domains in PIF3. The amino acid of the conserved site has been marked above the corresponding column. 


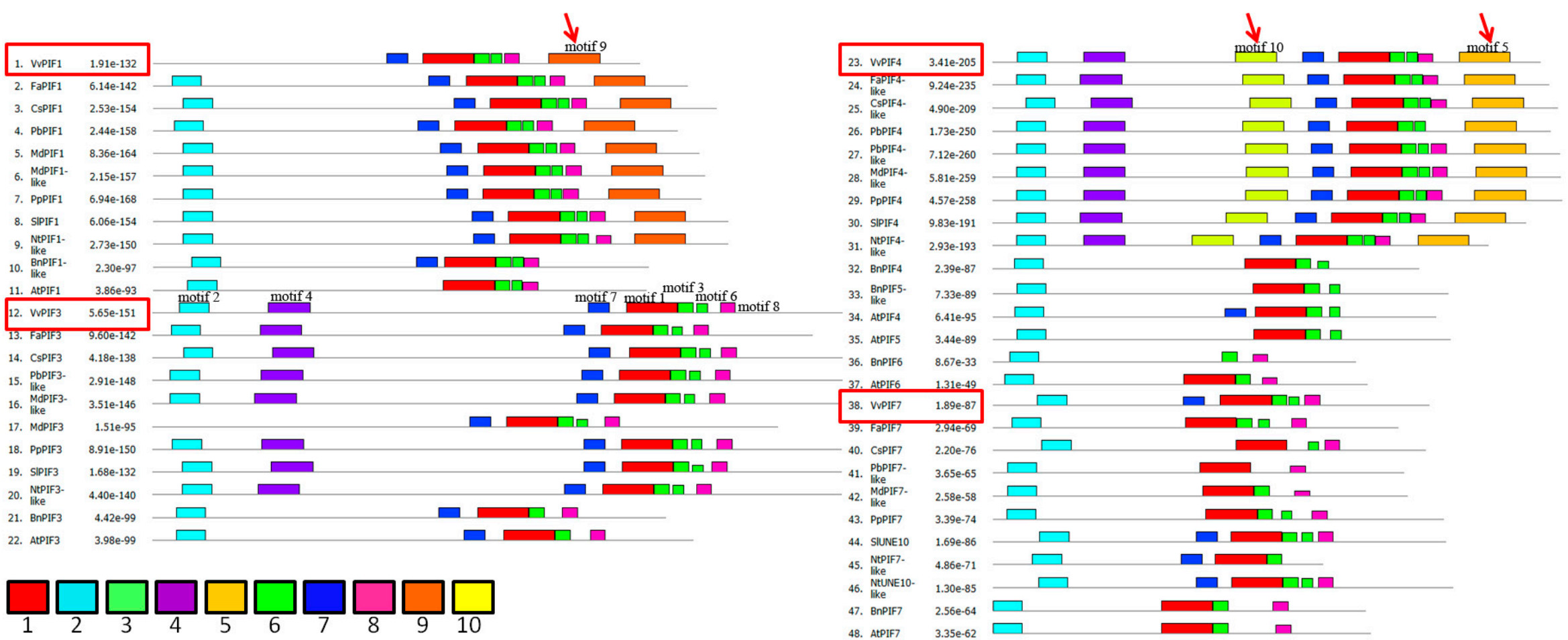

Figure 4. MEME analysis of the conserved motifs of PIFs protein. The motif number was set as 10 and one color corresponds to one motif. The amino acid sequence corresponding to each motif was present in Figure S1. The arrows indicate the specific motifs for PIF1 and PIF4 compared to other PIFs. The motif name of each color was labeled in VvPIF1, VvPIF3 and VvPIF4, respectively. 


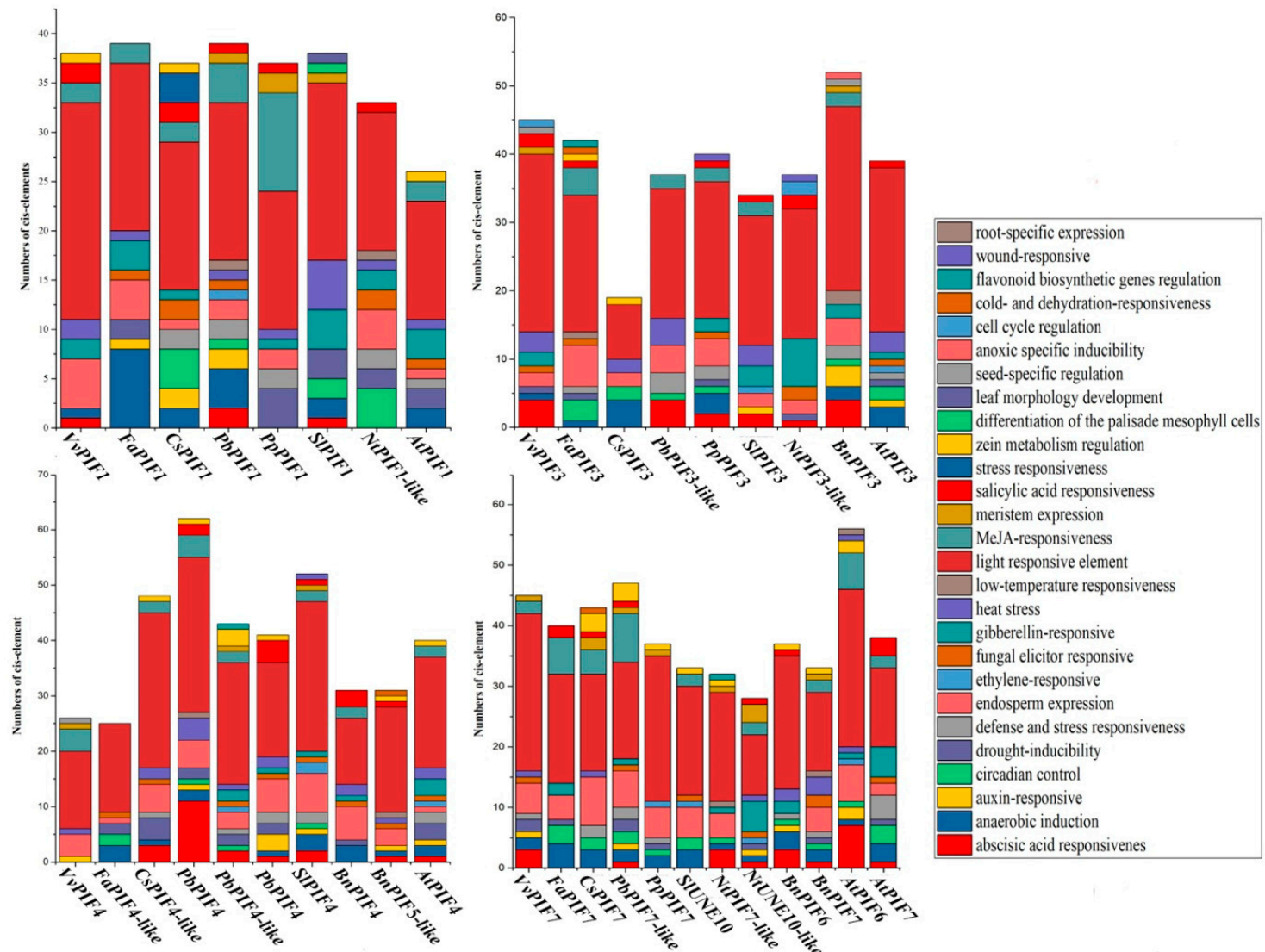

Figure 5. Cis-elements in the promoter of PIFs genes that are related to stress responses and plant development.

\subsection{Expression Profiles of PIFs in Different Organs}

There are differences in the expression profiles of PIFs in grape organs (Figure 6). The transcriptional level of VvPIF4 was the highest in roots, leaves, skin, and flesh, while VvPIF7 showed the highest expression in stems, tendrils, and flowers. According to the Figure 6, VvPIF1 and VvPIF3 expressed higher in leaves than that in other tissues.

According to the transcriptome data of Corvina (Figure S3), the VvPIF4 highly expressed in all tissues, except in the buds at germination stage (Bud-B, Bud-AB). VvPIF3 was at higher transcriptional levels than VvPIF4 and VvPIF7 in most organs, such as the berries in stages before maturation (Berry Pericarp-FS, Berry Pericarp-PFS, Berry Pericarp-V), buds during sprouting and developing (Bud-S, Bud-B, Bud-L, Bud-W), the growing flower (Flower-FB, Flower-F), leaves before senescence (Leaf-Y, Leaf-FS), the developing rachis (Rachis-FS, Rachis-PFS), stems and tendril (Stem-G, Stem-W, Tendril-Y, Tendril-WD, Tendril-FS). Compared with the expression of other PIFs, PIF7 was only expressed higher at Bud-B, Leaf-Y, Leaf-FS and Tendril, and the transcription level of PIF1 was low in most organs.

When comparing the transcriptional data of PIFs between SB and Corvina, the transcriptional levels of PIF4 were similar and high in roots, mature leaves, and fruits (skin and flesh) of both varieties. But the relative level of PIF7 was a little different in the young stem, tendril and inflorescence of these two cultivars, and the transcription level of PIF7 in Corvina was not as high as that in SB. These differences may be related to the cultivar characteristic and developmental stage. Taken together, the transcription level of PIF4 was the highest in most organs of the grape, followed by PIF3 and PIF7. 


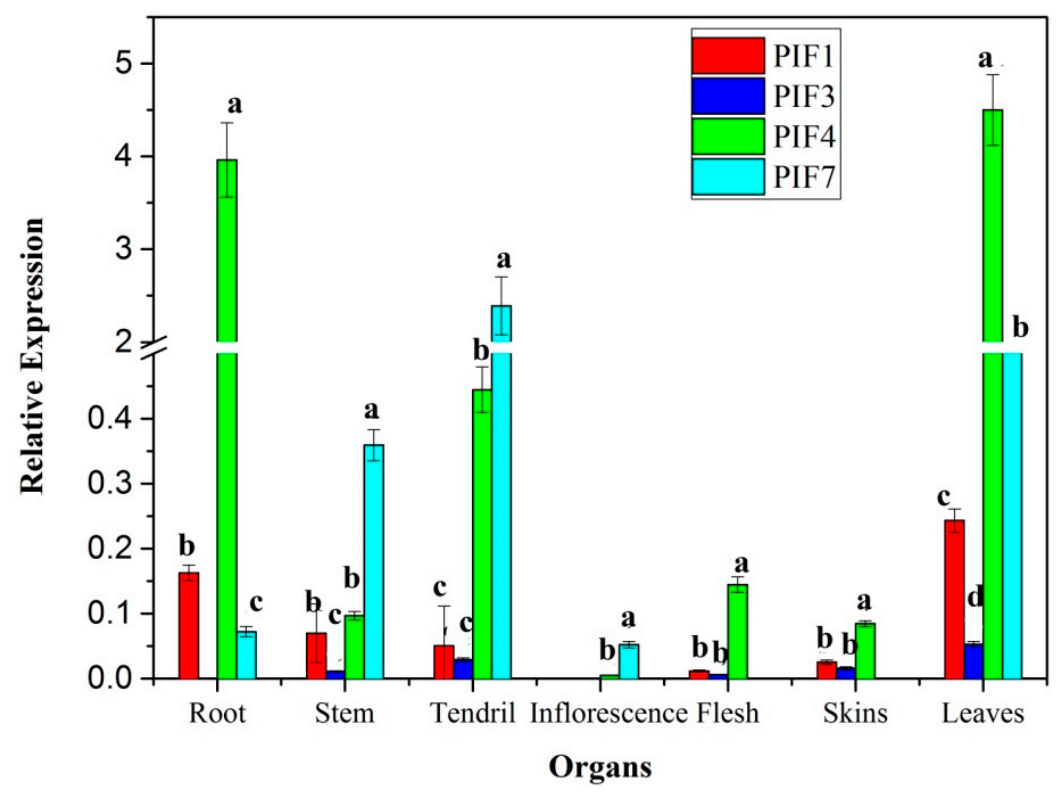

Figure 6. Relative expression levels of Vinitus vinifera phytochrome-interacting factors (VvPIFs) in different organs of Summer Black (SB). Values were normalized against the expression data of $V v U B I$ gene and are given as means \pm standard error among three biological replicates. Different letters indicate significant differences between genes $(p<0.05)$ according to Duncan's multiple test. The expression levels were calculated based on the $2^{-\Delta \mathrm{Ct}}$ method.

\subsection{Expression Profiles of PIFs During Grape Berries Development}

The transcriptional level of PIFs during fruit development showed a different pattern in different colored grape varieties (Figure 7). In SB, the transcriptional levels of PIF1 in grape berries slightly changed, except the short-term peaks appearing in the flesh during DS1 and in the skin during DS2. The transcriptional level of PIF3 kept stable in the skin and decreased significantly in the flesh. The expression of PIF4 and PIF7 firstly increased and then decreased in the skin, whereas they showed overall downward trends in the flesh. In short, except the PIF3 in the skin, the expression level of PIFs in SB showed a first increasing and then decreasing trend in the skin and an overall downward trend in the flesh. In SM, the expression of PIF1 showed an overall increasing trend in skin and a fluctuating pattern in the flesh. The expression of PIF3 showed a trend of first increasing and then decreasing both in the skin and flesh. The expression of PIF4 and PIF7 showed an overall upward trend in the skin and downward trend in the flesh. In short, except the PIF3 in the skin, the expression level of PIFs in SM showed an increasing trend in the skin and an overall downward trend in the flesh. The expression patterns of PIFs in the flesh of the two grape varieties were similar, indicating that the coloration of the skin did not affect the rhythmic expression pattern of PIFs in the flesh. 

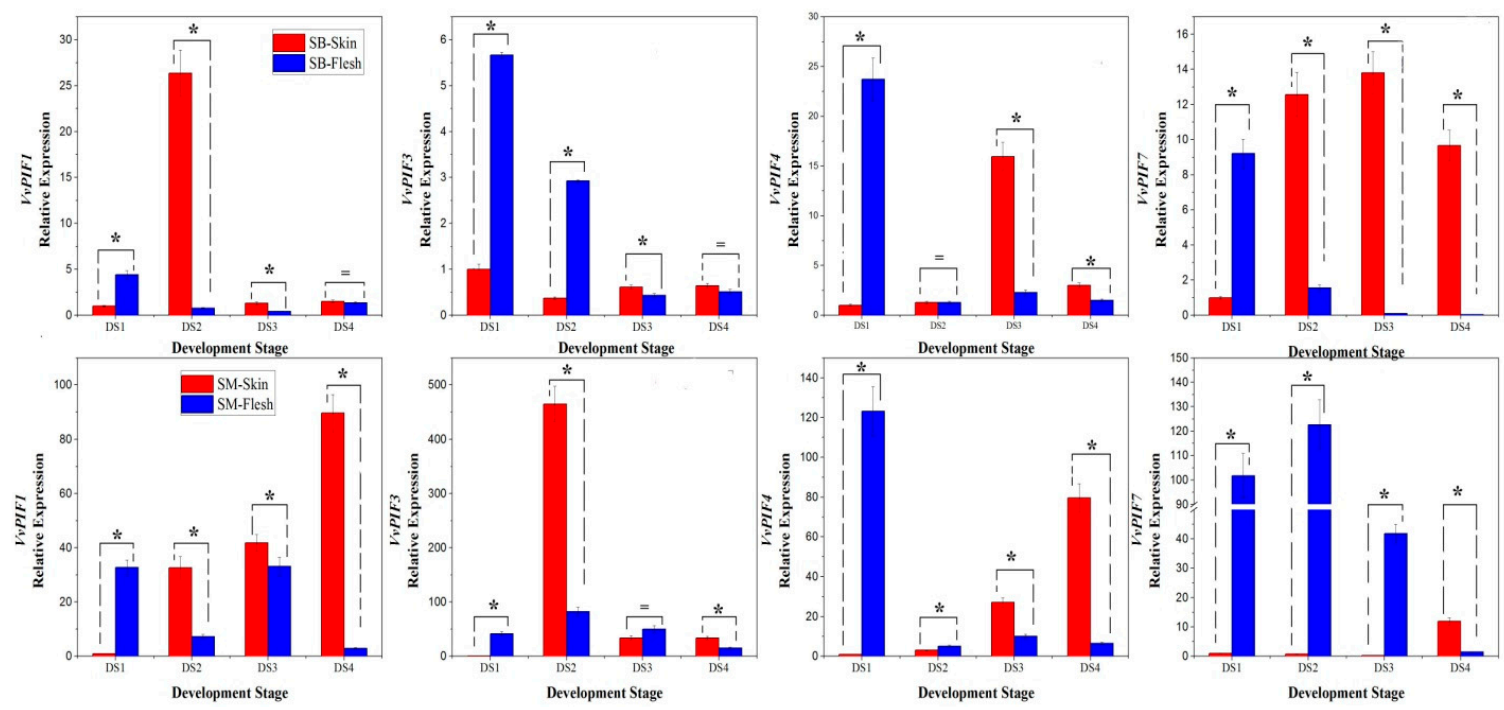

Figure 7. Relative expression levels of VvPIFs in the skin and flesh of SB (top panels) and Shine Muscat (SM; bottom panels) berries during different developmental stages (DS). Stars indicate significant differences between skin and flesh $(p<0.05)$ according to Duncan's multiple test, while the equal sign means non-specific difference. DS1, DS2, DS3, DS4 represent the early stage of fruit enlargement, pre-verasion, post-verasion and maturity respectively. The skin of SB and SM were purple-black and yellow-green, respectively. Due to the low expression values of PIFs in these organs, expression levels were calculated based on the $2^{-\Delta \Delta \mathrm{Ct}}$ method with the first stage sample chosen as a reference for convenience of observation.

\subsection{Expression Profiles of PIFs under Different Treatments}

The purpose of the lighting/shading experiment in leaves was to investigate the sensitivity of PIFs to light and dark conditions at the transcriptional level. The test was carried out in a thermostatic artificial climate chamber. A total of $12 \mathrm{~h}$ of light treatment, $12 \mathrm{~h}$ of dark treatment, and $12 \mathrm{~h}$ of light and dark alternate treatment were designed. Zero hours represented the starting point of the test, $12 \mathrm{~h}$ represented the end of the test, and $6 \mathrm{~h}$ was especially important for shading-lighting treatment, representing the time of light and dark alternating. From 0 to $6 \mathrm{~h}$, the expression of PIF1 in grape leaves showed a decreasing trend under light conditions, and a fluctuating trend under shading conditions, with peaks appearing at 2 and $6 \mathrm{~h}$ (Figure 8). From $8 \mathrm{~h}$ to $12 \mathrm{~h}$, the expression of PIF1 increased both under light and shading, but changed slightly under shading-lighting conditions. Notably, the increasing trend under shading conditions was more obvious. These results indicated that dark treatment was beneficial to increase the expression level of PIF1, and sudden light treatment had an inhibitory effect on the intrinsic expression pattern of PIF1. Shading didn't change the PIF1 overall changing patterns. From 0 to $6 \mathrm{~h}$, the expression level of PIF3 was close in shading and light conditions except for $2 \mathrm{~h}$, However, at $10 \mathrm{~h}$, the expression level of PIF3 under dark conditions showed a clear peak, extremely higher than that under other treatments. The expression level of PIF4 decreased from 0 to $6 \mathrm{~h}$ under shading and lighting conditions. Similar to the expression changes of PIF3, PIF4 also showed a significant peak at $10 \mathrm{~h}$. These results indicated that shading was beneficial to increase the expression of PIF3 and PIF4 in leaves, but the effect of shading was limited by circadian or the length of shading time. The expression level of PIF7 was low and changed gently under light conditions from $0-6 \mathrm{~h}$, while that showed a clear peak at $2 \mathrm{~h}$ and then decreased rapidly. From 6 to 12 $\mathrm{h}$, the transcription level of PIF7 under light treatment increased first and then decreased, while that under shading treatment showed a similar trend but changed more obviously. The expression level of PIF7 in the shading-lighting treatment showed a continuous downward trend, indicating that sudden light conditions also had an inhibitory effect on the intrinsic expression pattern of PIF7. Leaf shading experiments showed that VvPIFs were sensitive to light changes. Shading treatment was beneficial to 
increase the expression level of PIF1 and PIF7, but didn't change its internal change pattern. Shading treatment also helped to increase the expression level of PIF3 and PIF4, but the enhancement effect was time limited. In addition to PIF3, sudden light treatment after shading conditions inhibited the expression of PIFs.
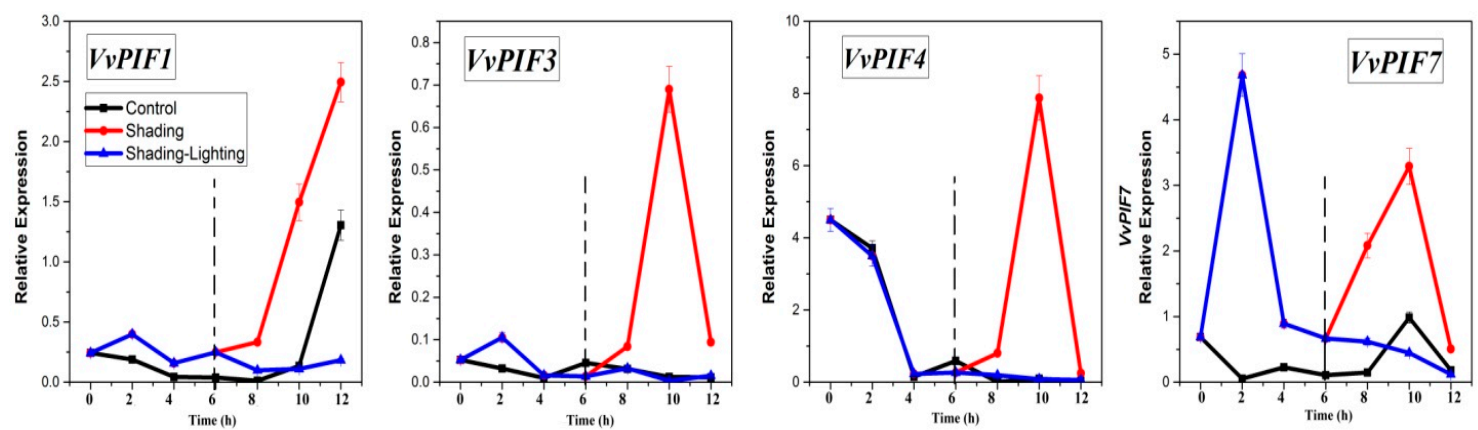

Figure 8. Relative expression levels of VvPIFs in the leaves of SB under light/shading conditions. The expression levels were calculated based on the $2^{-\Delta C t}$ method.

Fruit shading shed different effects on the expression patterns of PIFs in the skin and flesh (Figure 9). Compared with that in the control berries, the transcriptional level of PIF1 of shading berries was higher both in the skin and flesh at maturity, which indicated that the shading treatment improved the expression levels of PIF1 in grape berries at maturity. According to this analysis method, it could be found that the shading treatment improved the transcriptional level of PIF3 in the skin only at $45 \mathrm{~d}$, while it promoted the level of PIF3 continuously in the flesh. Contrary to the change in transcription patterns of PIF3, the transcriptional level of PIF4 in the skin was reduced at $45 \mathrm{~d}$ and showed a constantly decreased trend in the flesh. After shading, the expression level of PIF7 in the skin was improved at $60 \mathrm{~d}$, but showed no significant change in the flesh at that time. It was PIF1, PIF3, PIF4, and PIF7 in the skin and PIF1, PIF3, and PIF4 in the flesh that were sensitive to light/shading changes.
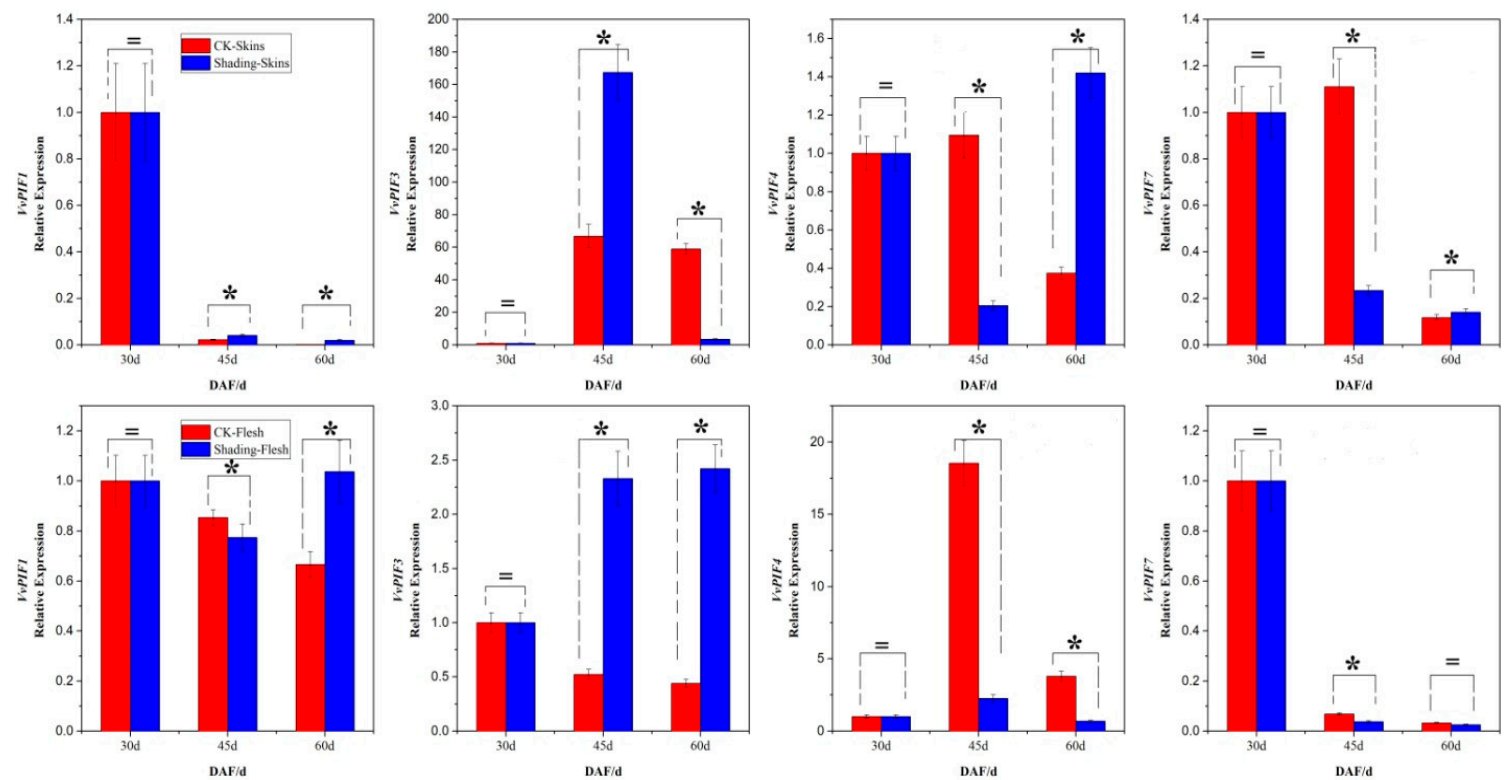

Figure 9. Relative expression levels of VvPIFs in the skin and flesh of SB under light/shading conditions. Stars indicate significant differences among different treatments $(p<0.05)$ according to Duncan's multiple test, while the equal sign means non-specific difference. DAF indicates days after flowering. Due to the low expression values of PIFs in these organs, expression levels were calculated based on the $2^{-\Delta \Delta \mathrm{Ct}}$ method with the first stage sample chosen as a reference for convenience of observation. 
The effects of different abiotic stresses on the expression levels of PIF4 and PIF7 were different. Salt and polyethylene glycol (PEG) treatment did not change the trend of PIF7 at different periods but increased the expression of PIF7 at 1 and $24 \mathrm{~h}$. The expression level of PIF4 gradually increased under PEG treatment. Under low temperature conditions, the transcriptional levels of PIF4 and PIF7 were down-regulated, but the latter decreased more significantly (Figure S4C,D). Under the long-time abiotic stresses, salt treatment did not alter the expression levels of PIF4 and PIF7 at D4, D8, and D12, but significantly increased their expression level at D16, indicating that salt can increase the transcription level of PIF4 and PIF7, without changing their developing patterns (Figure S4A,B). Under water deficit conditions, the transcription level of PIF7 and PIF4 was significantly increased at D4, D16, and at D12, D16 individually, indicating that water deficit has a delayed promoting effect on the expression levels of PIF4 and PIF7.

The transcription of PIF1, PIF3, PIF4 and PIF7 were detected in strawberry. The expression levels of PIF1 and PIF3 in fruit were high, while the expression levels of PIF4 and PIF7 were relatively low. After two weeks of fruit setting, the expression level of PIF1 and PIF3 in strawberry fruit showed a downward trend, and the expression of PIF4 and PIF7 first decreased and then increased. The expression of PIF1, PIF3, PIF4, and PIF7 was detected in mature fruit of citrus and the expression changes of PIFs were relatively stable, which may be due to the stability of endogenous metabolism in mature fruits. During the development of apple fruit, PIF1, PIF1-like, PIF3-like, PIF4-like, and PIF7-like all showed a gradually increasing trend. The expression of PIF3 first increased and then decreased, indicating the expression changes of PIFs were not uniform and there may be some differences in the function of PIFs in different types of fruit (Figure S5).

\section{Discussion}

Light is an important environmental factor that regulates the development and coloration of grape berries. Therefore, the study of PIFs, as an important light signal regulator, is expected to be an interesting entry point for exploring the relationship between light and the appearance quality and intrinsic quality of grape berries. Due to the differences in chromosome location, amino acid sequence, and promoter sequences, the PIFs in grapes showed great differences in transcriptional levels and the potential functions.

\subsection{PIFs Family and Their Evolutionary Analyses in Grapes}

We identified a total of four PIF genes PIF1, PIF3, PIF4, and PIF7 in the grape database, and they were distributed on four different chromosomes. By comparative alignment, many differences in the gene sequence and amino acid structure were detected. As gene exon-intron structures are typically conserved among homologous gene families [45], analysis of exon-intron structures will be helpful to reveal the evolutionary history of certain gene family [46].

From Figure 1, the PIF1 gene structure and the number of exons and introns are relatively conserved in different species, but the gene length varies greatly, which is similar to the findings of [47]. On the other hand, the genes structure of PIF3, PIF4, and PIF7 are not conserved in different plant species. Alterations in exon-intron structure within the coding region of a gene family might cause changes in their functions [48]. Compared with other fruit species, the gene lengths of VvPIF3 and VvPIF7 are longer. In grapes, PIF1, PIF3, and PIF4 all contain 7 exons, while PIF7 contains 6 exons. The second exon of each gene is the longest.

Phytochrome is an important functional protein that interacts with PIFs. The binding between phytochromes and PIFs depends on the correspondingly conserved sequences. Phytochrome-interacting factors have highly conserved APB motifs that are essential for PHYB binding [43]. AtPIF1 and AtPIF3 contain APA motifs respectively, which is also essential for the binding of PHYA [44,49]. Huq et al. [50] found that all AtPIFs have an APB motif and only PIF1, PIF3 have both an APB and an APA motif. In maize, there are at least seven putative PIFs with a conserved APB motif, of which two (ZmPIF3.1 and ZmPIF3.2) additionally also contain the APA motif, but only 
the interaction of ZmPIF3.1 and ZmPIF3.2 with ZmphyB1 has been proved now [51]. In grapes, the loss of the APB site in VvPIF1 may result in the inability of PHYB binding to this protein and affect the transmission of light signals. In terms of motifs in different species, PIF1, PIF3, and PIF4 are more conserved, and there are two specific motifs in PIF4 (Figure 4). Considering the existence of APB and APA [43,44,49,52], some conserved amino acids in motifs 10 and motifs 5 may be essential amino acids for maintaining the specific function of PIF4. PIF7 is less conserved in these species, and the function may be various in different plants.

Grapevines, like Arabidopsis and poplar, are dicotyledonous plants that diverged from monocotyledons about 130-240 million years ago [53]. Many dicotyledonous underwent whole genome triplication in recent million years, which made the duplications within PIF1, PIF7 and PIF8 clades in Solanum [54], grapevine genome has not undergone recent genome duplication, thus enabling the discovery of ancestral traits and features of the genetic organization of flowering plants. Combined with relative evolution time analysis, PIF7 has an earlier evolutionary time, and VvPIF7 may belong to the original PIF sequence. VvPIF4 was formed later and may have certain plant specificity. Based on the results of collinear analysis, the functional analysis of PIF1, PIF3, and PIF7 in model plants can contribute to the prediction of regulatory networks of corresponding VvPIFs.

\subsection{Function Analysis of the PIFs}

PIFs are important regulators of light signal transduction and each specific photoreaction can be regulated by several different PIFs [1,6,52]. PIF1 can inhibit the seeds germination. Both PIF1 and PIF3 promote hypocotyls negative gravitropism and inhibit the synthesis of chlorophyll. PIF4 and PIF5 can promote daily rhythmic growth of plants, and PIF7 can participate in enhancing shade avoidance of plants [1,52]. In recent years, researches on the synergies between PIF and other factors have gradually increased. Ni et al. [55] found that Photoregulatory Protein Kinases (PPKs) were collectively necessary for the normal light-induced phosphorylation and degradation of PIF3, and are critical components of a transcriptionally centered signaling hub that pleiotropically regulates plant growth and development in response to multiple signaling pathways. Kim et al. [56] identified the Repressor of Photosynthetic Genes1 (RPGE1) as a direct target of PIF1, which acted downstream of PIF1 in the endodermis to repress photosynthetic genes and regulate plastid development.

To clarify the metabolic network of PIFs in grapes, we performed promoter analysis and functional prediction of PIFs. The number of cis-elements related to photoreaction in the promoter of PIFs is the most abundant, which corresponds to the role of PIFs in the light response process.

ZmPIF1 promoter region was rich in drought response (MBS) and ABRE-related elements, and that the expression level of ZmPIF1 was significantly induced by drought and abscisic acid treatments [57]. In grapes, there is less cis-elements related to ABA response and no cis-elements related to drought response. The response of VvPIF1 to ABA and drought signals may not be as sensitive as ZmPIF1.

AtPIF3 is the main regulator promoting hypocotyls elongation in response to ethylene [9] and there is an ethylene response related element in its promoter. However, many ABA, instead of ethylene, responding related elements were found in the promoter of VvPIF3, which indicated that grapes may react differently from Arabidopsis in response to ABA and ethylene stimulation. PIF4 is the main regulator promoting hypocotyl elongation and early flowering response under high temperature $[17,58]$. There are only heat stress elements and no low-temperature response elements in the upstream of VvPIF4. VvPIF4 was transcribed at low levels under the low temperature stress and might participate in the regulation of growth under high temperature. The transcription levels of VvPIF4 and VvPIF7 both increased under drought stress, but drought response elements were only detected in the promoter of VvPIF7, not in VvPIF4. VvPIF7 and VvPIF4 may respond to drought signals in different regulatory ways.

HY5 is a basic leucine zipper (bZIP) transcription factor that can form dynamic activation-suppression transcription module with PIFs, which directly act on the promoter cis-element G-box to cope with light and temperature changes. In regulating photosynthetic pigment synthesis 
genes, HY5 and PIFs do not operate alone, but with the circadian clock [59]. Through prediction of protein function, VvPIF1 may also interact with VvHY5 protein and participate in the regulation of related metabolic activities. GAI is a kind of DELLA protein, could function as negative modulators of PIF protein transcriptional regulatory activity by inhibiting their DNA-binding activities [60], The function of VvPIF3 and VvPIF4 may also be regulated by GAI protein.

SIPIF1 a were identified in tomatoes. Upon induction of ripening, PIF1a transcript levels increased approximately fivefold in red ripe fruit compared to green samples [27]. In grapes, the expression pattern of PIF1 in the skin of SM was similar to SIPIF1a (Figure 7), but that in the skin of SB was so different. These difference may be related to the accumulation of anthocyanins in SB skin, which were hardly synthesized in the skin of tomatoes and SM. Additionally, in the two grape cultivars, the transcription levels of PIF4 and PIF7 increased in the skin during the period before maturation while the expression of all PIFs generally showed a downward trend in the flesh, which indicated that the skin coloration didn't affect or showed little effect on the expression of genes in the flesh at the transcriptional level. At the same time, after the shading treatment, the transcription level of PIFs in the skin and flesh of the two cultivars changed greatly, indicating that artificially changing the external light environment could directly or indirectly cause changes in the expression pattern of PIFs in the skin and flesh.

In Arabidopsis, the expression of PIF4/5 was repressed during the beginning of the dark period and rose in the middle of the night to peak at dawn, whereas the transcript levels PIF3, and possibly PIF1, were relatively constant [61-63]. In grape leaves, for the shading treatment (Figure 8, red line from $2 \mathrm{~h}$ to $10 \mathrm{~h}$ ), the expression levels of PIF1, PIF3, PIF4, PIF7 all generally showed a firstly decreasing and then increasing trend, similar to the patterns of AtPIF4 and AtPIF5. The specific expression pattern of PIF1 and PIF3 in grape, and the repression of PIF3, PIF4 and PIF7 expression at the end of dark treatment may be related to various factors. On the one hand, the test conditions of Arabidopsis and grapes were different. For example, the tinfoil was used to simulate the dark conditions of leaves in this study, but the whole plant was not treated darkly. On the other hand, Arabidopsis belongs to the herb while the grapes belong to the wood. Different plants have large differences in photomorphogenesis mechanisms, so the role of PIFs in plants, their expression patterns, and their sensitivity to light and darkness were also various.

Leaf shading experiments showed that VvPIFs were sensitive to light changes and shading treatment was beneficial to increase the expression level of PIFs though their reaction time was not the same. Recently, the function of PIFs in fruit trees has begun to be identified and functionally studied. Zhou et al. [47] identified the PIF1 in apple and found the PIF1 was not only involved in the germination of apple seeds and dormancy breaking of apple buds, but also inhibited the apple calli via PHY-mediated pathways. The function of various PIFs in grapes still needs further studies.

\section{Conclusions}

We identified PIF1, PIF3, PIF4, and PIF7 in the grapevine. These members are distributed on four different chromosomes with similar gene structures. VvPIFs (except VvPIF4) were located at the syntenic block with those from A. thaliana, S. lycopersicum, or C. sinensis. PIF3, PIF4, and PIF7 in grapes have conserved APB sequences. VvPIF1 has a conserved PIF1-specific APA sequence, while amino acid mutations occur in the specific APA sequences in VvPIF3. What's more, two specific motifs are found in the PIF4 amino sequence. The photoreceptor-related elements in the VvPIFs promoter region are the most abundant. VvPIF1 and VvHY5, VvPIF3, VvPIF4 and VvGAI1 may interact with each other and participate in light signal transduction together.

The relative expression levels of VvPIFs varied in different organs at different developmental stages, PIF4 was expressed at the highest level in most organs of grapevines. The transcriptional levels of PIF4 and PIF7 increased in the skin while the expression of all PIFs generally showed a downward trend in the flesh during the period before maturation. The transcription level of PIFs in grape leaves was sensitive to light/dark changes. Shading treatment was beneficial to enhance the transcription 
level of VvPIFs, but the effect is time-controlled on VvPIF3 and VvPIF4. These findings could lay the theoretical foundation for the function study of PIFs and the further construction of grape light regulation networks.

Supplementary Materials: The following are available online at http:/ /www.mdpi.com/2073-4425/9/9/451/s1, Table S1. Synteny regions of FIFs genes between grape and Arabidopsis thaliana, Solanum lycopersicum, Citrus sinensis, Figure S1. The amino acid sequence of PIFs motifs in MEME analysis, Figure S2. The interaction protein prediction of VvPIFs, Figure S3. Expression patterns of the grape PIFs family in different organs of 'Corvina', Figure S4. Expression patterns of the grape PIFs family in the leaves of 'Cabernet Sauvignon' under long or short term abiotic stress, Figure S5. Expression patterns of the PIFs family in the fruits of strawberry, citrus, and apple, Table S2. List of primer sequences used in QPCR analysis, Table S3. Prediction functional partners of PIFs and their annotation.

Author Contributions: Conceptualization, H.J. and J.F., Data curation, X.Z., Formal analysis, K.Z. and T.Z.; Funding acquisition, J.F., Investigation, K.Z. and T.Z.; Methodology, X.Z. and Z.L.; Software, H.J.; Supervision, J.F.; Validation, S.J.; Visualization, S.J.; Writing—original draft, K.Z.; Writing—review \& editing, L.G.

Funding: This research was funded by the Natural Science Foundation of China (NSFC; No. 31672131), the earmarked fund for Jiangsu Agricultural Industry Technology System (SXGC [2017]278), and Jiangsu Major Species Creation Project (PZCZ201724).

Acknowledgments: We thank Shaofang He, and Chuanyun Cheng for help in syntenic analysis and vineyard management.

Conflicts of Interest: The authors declare no conflict of interest.

\section{References}

1. Pham, V.N.; Kathare, P.K.; Huq, E. Phytochromes and phytochrome interacting factors. Plant Physiol. 2018, 176, 1025-1038. [CrossRef] [PubMed]

2. Bae, G.; Choi, G. Decoding of light signals by plant phytochromes and their interacting proteins. Annu. Rev Plant Biol. 2008, 59, 281-311. [CrossRef] [PubMed]

3. Rockwell, N.C.; Su, Y.S.; Lagarias, J.C. Phytochrome structure and signaling mechanisms. Annu. Rev Plant Biol. 2006, 57, 837-858. [CrossRef] [PubMed]

4. Lee, N.; Choi, G. Phytochrome-interacting factor from Arabidopsis to liverwort. Curr. Opin. Plant Biol. 2017, 5, 54-60. [CrossRef] [PubMed]

5. Possart, A.; Xu, T.; Paik, I.; Hanke, S.; Keim, S.; Hermann, H.M.; Wolf, L.; Hiss, M.; Becker, C.; Huq, E.; et al. Characterization of phytochrome interacting factors from the moss Physcomitrella patens illustrates conservation of phytochrome signaling modules in land plants. Plant Cell 2017, 29, 310-330. [CrossRef] [PubMed]

6. Leivar, P.; Quail, P.H. PIFs: Pivotal components in a cellular signaling hub. Trends Plant Sci. 2011, 16, 19-28. [CrossRef] [PubMed]

7. Oh, E.; Kim, J.; Park, E.; Kim, J.I.; Kang, C.; Choi, G. PIL5, a phytochrome-interacting basic helix-loop-helix protein, is a key negative regulator of seed germination in Arabidopsis thaliana. Plant Cell 2004, 16, 3045-3058. [CrossRef] [PubMed]

8. Moon, J.; Zhu, L.; Shen, H.; Huq, E. PIF1 directly and indirectly regulates chlorophyll biosynthesis to optimize the greening process in Arabidopsis. Proc. Natl. Acad. Sci. USA 2008, 105, 9433-9438. [CrossRef] [PubMed]

9. Zhong, S.; Shi, H.; Xue, C.; Wang, L.; Xi, Y.; Li, J.; Quail, P.H.; Deng, X.W.; Guo, H. A molecular framework of light-controlled phytohormone action in Arabidopsis. Curr. Biol. 2012, 22, 1530-1535. [CrossRef] [PubMed]

10. Monte, E.; Tepperman, J.M.; Al-Sady, B.; Kaczorowski, K.A.; Alonso, J.M.; Ecker, J.R.; Li, X.; Zhang, Y.; Quail, P.H. The phytochrome-interacting transcription factor, PIF3, acts early, selectively, and positively in light-induced chloroplast development. Proc. Natl. Acad. Sci. USA 2004, 101, 16091-16098. [CrossRef] [PubMed]

11. Shin, J.; Park, E.; Choi, G. PIF3 regulates anthocyanin biosynthesis in an HY5-dependent manner with both factors directly binding anthocyanin biosynthetic gene promoters in Arabidopsis. Plant J. 2007, 49, 981-994. [CrossRef] [PubMed] 
12. Jiang, B.; Shi, Y.; Zhang, X.; Xin, X.; Qi, L.; Guo, H.; Li, J.; Yang, S. PIF3 is a negative regulator of the CBF pathway and freezing tolerance in Arabidopsis. Proc. Natl. Acad. Sci. USA 2017, 114, E6695-E6702. [CrossRef] [PubMed]

13. Lee, C.M.; Thomashow, M.F. Photoperiodic regulation of the C-repeat binding factor (CBF) cold acclimation pathway and freezing tolerance in Arabidopsis thaliana. Proc. Natl. Acad. Sci. USA 2012, 109, 15054-15059. [CrossRef] [PubMed]

14. Sakuraba, Y.; Jeong, J.; Kang, M.Y.; Kim, J.; Paek, N.C.; Choi, G. Phytochrome-interacting transcription factors PIF4 and PIF5 induce leaf senescence in Arabidopsis. Nat. Commun. 2014, 5, 4636. [CrossRef] [PubMed]

15. Zhu, J.Y.; Oh, E.; Wang, T.; Wang, Z.Y. TOC1-PIF4 interaction mediates the circadian gating of thermoresponsive growth in Arabidopsis. Nat. Commun. 2016, 7, 13692. [CrossRef] [PubMed]

16. Lorrain, S.; Allen, T.; Duek, P.D.; Whitelam, G.C.; Fankhauser, C. Phytochrome-mediated inhibition of shade avoidance involves degradation of growth-promoting bHLH transcription factors. Plant J. 2008, 53, 312-323. [CrossRef] [PubMed]

17. Franklin, K.A.; Lee, S.H.; Patel, D.; Kumar, S.V.; Spartz, A.K.; Gu, C.; Ye, S.; Yu, P.; Breen, G.; Cohen, J.D.; et al. Phytochrome-interacting factor 4 (PIF4) regulates auxin biosynthesis at high temperature. Proc. Natl. Acad. Sci. USA 2011, 108, 20231-20235. [CrossRef] [PubMed]

18. Liu, Z.; Zhang, Y.; Wang, J.; Li, P.; Zhao, C.; Chen, Y.; Bi, Y. Phytochrome-interacting factors PIF4 and PIF5 negatively regulate anthocyanin biosynthesis under red light in Arabidopsis seedlings. Plant Sci. 2015, 238, 64-72. [CrossRef] [PubMed]

19. Penfield, S.; Josse, E.M.; Halliday, K.J. A role for an alternative splice variant of PIF6 in the control of Arabidopsis primary seed dormancy. Plant Mol. Biol. 2010, 73, 89-95. [CrossRef] [PubMed]

20. Li, L.; Ljung, K.; Breton, G.; Schmitz, R.J.; Pruneda-Paz, J.; Cowing-Zitron, C.; Cole, B.J.; Ivans, L.J.; Pedmale, U.V.; Jung, H.S.; et al. Linking photoreceptor excitation to changes in plant architecture. Genes Dev. 2012, 26, 785-790. [CrossRef] [PubMed]

21. Leivar, P.; Monte, E.; Al-Sady, B.; Carle, C.; Storer, A.; Alonso, J.M.; Ecker, J.R.; Quail, P.H. The Arabidopsis phytochrome-interacting factor PIF7, together with PIF3 and PIF4, regulates responses to prolonged red light by modulating phyB levels. Plant Cell 2008, 20, 337-352. [CrossRef] [PubMed]

22. Oh, E.; Zhu, J.Y.; Wang, Z.Y. Interaction between BZR1 and PIF4 integrates brassinosteroid and environmental responses. Nat. Cell Biol. 2012, 14, 802. [CrossRef] [PubMed]

23. Zhang, D.; Jing, Y.; Jiang, Z.; Lin, R. The chromatin-remodeling factor PICKLE integrates brassinosteroid and gibberellin signaling during skotomorphogenic growth in Arabidopsis. Plant Cell 2014, 26, 2472-2485. [CrossRef] [PubMed]

24. Toledo-Ortiz, G.; Huq, E.; Rodríguez-Concepción, M. Direct regulation of phytoene synthase gene expression and carotenoid biosynthesis by phytochrome-interacting factors. Proc. Natl. Acad. Sci. USA 2010, 107, 11626-11631. [CrossRef] [PubMed]

25. Sheehan, M.J.; Farmer, P.R.; Brutnell, T.P. Structure and expression of maize phytochrome family homeologs. Genetics 2004, 167, 1395-1405. [CrossRef] [PubMed]

26. Li, F.W.; Melkonian, M.; Rothfels, C.J.; Villarreal, J.C.; Stevenson, D.W.; Graham, S.W.; Wong, G.K.; Pryer, K.M.; Mathews, S. Phytochrome diversity in green plants and the origin of canonical plant phytochromes. Nat. Commun. 2015, 6, 7852. [CrossRef] [PubMed]

27. Llorente, B.; D’andrea, L.; Ruiz-Sola, M.A.; Botterweg, E.; Pulido, P.; Andilla, J.; Loza-Alvarez, P.; Rodriguez-Concepcion, M. Tomato fruit carotenoid biosynthesis is adjusted to actual ripening progression by a light-dependent mechanism. Plant J. 2016, 85, 107-119. [CrossRef] [PubMed]

28. Phoenix Bioinformatics Corporation. The Arabidopsis Information Resource (TAIR) Database. Available online: https://www.arabidopsis.org/portals/education/aboutarabidopsis.jsp (accessed on 6 September 2018).

29. Genoscope. Grapevine Genome Browser. Available online: http://www.genoscope.cns.fr/externe/ GenomeBrowser/Vitis/ (accessed on 6 September 2018).

30. Grape Genome Database, CRIBI, Version 2.1. Available online: http://genomes.cribi.unipd.it/grape/ (accessed on 6 September 2018).

31. Zhu, X.; Wang, M.; Li, X.; Jiu, S.; Wang, C.; Fang, J. Genome-wide analysis of the sucrose synthase gene family in grape (Vitis vinifera): Structure, evolution, and expression profiles. Genes 2017, 8, 111. [CrossRef] [PubMed] 
32. ELIXIR. InterProScan. Available online: http://www.ebi.ac.uk/Tools/pfa/iprscan5/ (accessed on 6 September 2018).

33. NCBI. The Conserved Domain Database, CDD. Available online: http://www.ncbi.nlm.nih.gov/cdd (accessed on 6 September 2018).

34. ExPasy. ProtParam Tool. Available online: http://web.expasy.org/protparam/ (accessed on 6 September 2018).

35. Song, J.; Zhou, Y.; Zhang, J.; Zhang, K. Structural, expression and evolutionary analysis of the non-specific phospholipase C gene family in Gossypium hirsutum. BMC Genom. 2017, 18, 979. [CrossRef] [PubMed]

36. Hu, B.; Jin, J.; Guo, A.-Y.; Zhang, H.; Luo, J.; Gao, G. GSDS 2.0: An upgraded gene feature visualization server. Bioinformatics 2015, 31, 1296-1297. [CrossRef] [PubMed]

37. Tamura, K.; Stecher, G.; Peterson, D.; Filipski, A.; Kumar, S. MEGA6: Molecular evolutionary genetics analysis version 6.0. Mol. Biol. Evol. 2013, 30, 2725-2729. [CrossRef] [PubMed]

38. Li, Z.; Zhang, C.; Guo, Y.; Niu, W.; Wang, Y.; Xu, Y. Evolution and expression analysis reveal the potential role of the HD-Zip gene family in regulation of embryo abortion in grapes (Vitis vinifera L.). BMC Genom. 2017, 18, 744. [CrossRef] [PubMed]

39. Wang, Y.; Tang, H.; DeBarry, J.D.; Tan, X.; Li, J.; Wang, X.; Lee, T.H.; Jin, H.; Marler, B.; Guo, H.; et al. MCScanX: A toolkit for detection and evolutionary analysis of gene synteny and collinearity. Nucleic Acids Res. 2012, 40, e49. [CrossRef] [PubMed]

40. Krzywinski, M.I.; Schein, J.E.; Birol, I.; Connors, J.; Gascoyne, R.; Horsman, D.; Jones, S.J.; Marra, M.A. Circos: An Information Aesthetic for Comparative Genomics. Genome Res. 2009, 2009 19, 1639-1645. [CrossRef]

41. Lescot, M.; Déhais, P.; Thijs, G.; Marchal, K.; Moreau, Y.; Van de Peer, Y.; Rouzé, P.; Rombauts, S. PlantCARE, a database of plant cis-acting regulatory elements and a portal to tools for in silico analysis of promoter sequences. Nucleic Acids Res. 2002, 30, 325-327. [CrossRef] [PubMed]

42. Wang, C.; Han, J.; Shangguan, L.; Yang, G.; Kayesh, E.; Zhang, Y.; Leng, X.; Fang, J. Depiction of grapevine phenology by gene expression information and a test of its workability in guiding fertilization. Plant Mol. Biol. 2014, 32, 1070-1084. [CrossRef]

43. Khanna, R.; Huq, E.; Kikis, E.A..; Al-Sady, B.; Lanzatella, C.; Quail, P.H. A novel molecular recognition motif necessary for targeting photoactivated phytochrome signaling to specific basic helix-loop-helix transcription factors. Plant Cell 2004, 16, 3033-3044. [CrossRef] [PubMed]

44. Al-Sady, B.; Ni, W.; Kircher, S.; Schäfer, E.; Quail, P.H. Photoactivated phytochrome induces rapid PIF3 phosphorylation prior to proteasome-mediated degradation. Mol. Cell 2006, 23, 439-446. [CrossRef] [PubMed]

45. Frugoli, J.A.; McPeek, M.A.; Thomas, T.L.; McClung, C.R. Intron loss and gain during evolution of the catalase gene family in angiosperms. Genetics 1998, 149, 355-365. [PubMed]

46. Lecharny, A.; Boudet, N.; Gy, I.; Aubourg, S.; Kreis, M. Introns in, introns out in plant gene families: A genomic approach of the dynamics of gene structure. J. Struct. Funct. Genom. 2003, 3, 111-116. [CrossRef]

47. Zhou, L.J.; Mao, K.; Qiao, Y.; Jiang, H.; Li, Y.Y.; Hao, Y.J. Functional identification of MdPIF1 as a Phytochrome Interacting Factor in Apple. Plant Physiol. Biochem. 2017, 119, 178-188. [CrossRef] [PubMed]

48. Xu, G.; Guo, C.; Shan, H.; Kong, H. Divergence of duplicate genes in exon-intron structure. Proc. Natl. Acad. Sci. USA 2012, 109, 1187-1192. [CrossRef] [PubMed]

49. Shen, H.; Zhu, L.; Castillon, A.; Majee, M.; Downie, B.; Huq, E. Light-induced phosphorylation and degradation of the negative regulator PHYTOCHROME-INTERACTING FACTOR1 from Arabidopsis depend upon its direct physical interactions with photoactivated phytochromes. Plant Cell 2008, 20, 1586-1602. [CrossRef] [PubMed]

50. Huq, E.; Al-Sady, B.; Hudson, M.; Kim, C.; Apel, K.; Quail, P.H. Phytochrome-interacting factor 1 is a critical bHLH regulator of chlorophyll biosynthesis. Science 2004, 305, 1937-1941. [CrossRef] [PubMed]

51. Kumar, I.; Swaminathan, K.; Hudson, K.; Hudson, M.E. Evolutionary divergence of phytochrome protein function in Zea mays PIF3 signaling. J. Exp. Bot. 2016, 67, 4231-4240. [CrossRef] [PubMed]

52. Leivar, P.; Monte, E. PIFs: Systems integrators in plant development. Plant Cell 2014, 26, 56-78. [CrossRef] [PubMed]

53. Jaillon, O.; Aury, J.M.; Noel, B.; Policriti, A.; Clepet, C.; Casagrande, A.; Choisne, N.; Aubourg, S.; Vitulo, N.; Jubin, $\mathrm{C}$; et al. The grapevine genome sequence suggests ancestral hexaploidization in major angiosperm phyla. Nature 2007, 449, 463. [PubMed] 
54. Rosado, D.; Gramegna, G.; Cruz, A.; Lira, B.S.; Freschi, L.; de Setta, N.; Rossi, M. Phytochrome interacting factors (PIFs) in Solanum lycopersicum: Diversity, evolutionary history and expression profiling during different developmental processes. PLoS ONE 2016, 11, e0165929. [CrossRef] [PubMed]

55. Ni, W.; Xu, S.L.; González-Grandío, E.; Chalkley, R.J.; Huhmer, A.F.; Burlingame, A.L.; Wang, Z.Y.; Quail, P.H. PPKs mediate direct signal transfer from phytochrome photoreceptors to transcription factor PIF3. Nat. Commun. 2017, 8, 15236. [CrossRef] [PubMed]

56. Kim, K.; Jeong, J.; Kim, J.; Lee, N.; Kim, M.E.; Lee, S.; Kim, S.C.; Choi, G. PIF1 regulates plastid development by repressing photosynthetic genes in the endodermis. Mol. Plant 2016, 9, 1415-1427. [CrossRef] [PubMed]

57. Gao, Y.; Wu, M.; Zhang, M.; Jiang, W.; Ren, X.; Liang, E.; Zhang, D.; Zhang, C.; Xiao, N.; Li, Y.; et al. A Maize phytochrome-interacting factors Protein ZmPIF1 Enhances Drought Tolerance by Inducing Stomatal Closure and Improves Grain Yield in Oryza sativa. Plant Biotechnol. J. 2018. [CrossRef] [PubMed]

58. Kumar, S.V.; Lucyshyn, D.; Jaeger, K.E.; Alós, E.; Alvey, E.; Harberd, N.P.; Wigge, P.A. Transcription factor PIF4 controls the thermosensory activation of flowering. Nature 2012, 484, 242. [CrossRef] [PubMed]

59. Toledo-Ortiz, G.; Johansson, H.; Lee, K.P.; Bou-Torrent, J.; Stewart, K.; Steel, G.; Rodríguez-Concepción, M.; Halliday, K.J. The HY5-PIF regulatory module coordinates light and temperature control of photosynthetic gene transcription. PLoS Genet. 2014, 10, e1004416. [CrossRef] [PubMed]

60. Feng, S.; Martinez, C.; Gusmaroli, G.; Wang, Y.; Zhou, J.; Wang, F.; Chen, L.; Yu, L.; Iglesias-Pedraz, J.M.; Kircher, S.; et al. Coordinated regulation of Arabidopsis thaliana development by light and gibberellins. Nature 2008, 451, 475. [CrossRef] [PubMed]

61. Nozue, K.; Covington, M.F.; Duek, P.D.; Lorrain, S.; Fankhauser, C.; Harmer, S.L.; Maloof, J.N. Rhythmic growth explained by coincidence between internal and external cues. Nature 2007, 448, 358-361. [CrossRef] [PubMed]

62. Soy, J.; Leivar, P.; González-Schain, N.; Sentandreu, M.; Prat, S.; Quail, P.H.; Monte, E. Phytochrome-imposed oscillations in PIF3 protein abundance regulate hypocotyl growth under diurnal light/dark conditions in Arabidopsis. Plant J. 2012, 71, 390-401. [CrossRef] [PubMed]

63. Soy, J.; Leivar, P.; Monte, E. PIF1 promotes phytochrome-regulated growth under photoperiodic conditions in Arabidopsis together with PIF3, PIF4, and PIF5. J. Exp. Bot. 2014, 65, 2925-2936. [CrossRef] [PubMed] 